\title{
PORE SIZE DISTRIBUTION OF CARBON WITH DIFFERENT Probe MOLeCules
}

Atichat Wongkoblap*, Worapot Intomya, Warangkhana Somrup, Sorod Charoensuk, Supunnee Junpirom and Chaiyot Tangsathitkulchai

School of Chemical Engineering, Institute of Engineering,

Suranaree University of Technology, Nakhon Ratchasima, Thailand 30000

E-mail: atichat@sut.ac.th*,intomya@gmail.com,warangkana@hotmail.com

sorod_sut@hotmail.com, supunneejun@yahoo.com and

chaiyot@sut.ac.th

\section{ABSTRACT}

In this study, a Grand Canonical Monte Carlo simulation (GCMC) method is used to study the adsorption of different probe molecules on activated carbon, while the experimental tests are performed by using a Gravimetric Analyzer. In addition the simulation results together with the measured isotherm data are used for the determination of micropore size distribution. Nitrogen at $77 \mathrm{~K}$ and carbon dioxide at 273 and $300 \mathrm{~K}$ are proposed as molecular probes. The simulation results obtained for various pore sizes represent the structure of molecular probe packing in the individual pores at different pressures. The reconstructed adsorption isotherm obtained by using these results and a postulated pore size distribution (PSD) function is used to determine the PSD of activated carbon which provides the best match between the simulation isotherm and the experimental isotherm. The PSD obtained using the GCMC agrees very well with the Density Functional Theory (DFT) method. The PSD for carbon dioxide differs from that for nitrogen due to the molecular structure and size. The advantage of GCMC is that it can provide not only adsorption isotherm but also the snapshot that presents the mechanism inside the pore.

\section{KEYWORDS}

activated carbon, GCMC, monte carlo simulation, pore side distribution 


\section{Introduction}

Activated carbon is usually used in adsorption process due to its large micropore and mesopore volumes and high surface area. The characterization of porous carbon (in terms of pore size distribution) is necessary for the utilization and design of improved carbon to serve in several applications. For this purpose, argon and nitrogen are often used as probe adsorbates at their respective boiling points. However to characterize carbon of fine microporosity, carbon dioxide adsorption shows a good capability of probing the narrowest micropores at experimentally measurable pressures [1].

To model porous carbon, it is assumed to consist of pores of different widths. They are assumed to be either infinite slit pores [2]-[3] or infinite cylindrical pores [4]. However, the infinite pore model is too ideal to reflect the adsorption behavior of activated carbon whose length is finite [5] and it contains functional groups on the basal graphene layers [6]. Therefore a real carbon pore of finite length and carbon surfaces as graphene layers comprising of carbon atoms arranged in a hexagonal pattern [7] is used in this study to model adsorption behavior of nitrogen and carbon dioxide.

In this paper, the adsorption isotherms of nitrogen at $77 \mathrm{~K}$ and carbon dioxide at 273 and $300 \mathrm{~K}$ are obtained for pore widths of 6.3 to $30 \AA$ by a Grand Canonical Monte Carlo (GCMC) simulation. These widths represent the micropore and mesopore commonly observed in activated carbon. The simulation results will be compared against the experimental data obtained for Longan seed activated carbons (LAC) produced in our laboratory and a commercial activated carbon (CAC) produced from coconut shell. The PSD obtained from GCMC will be compared with that obtained from DFT method.

\section{Page Dimensions}

\subsection{Fluid model}

In this paper, carbon dioxide is modeled as a 3-center-Lennard-Jones (LJ) molecule having LJ interaction sites on the atoms and point charges to account for the quadrupole moment while nitrogen is modeled as a simple spherical LJ molecule. The molecular parameters for $\mathrm{CO}_{2}$ proposed by Harris and Yung [8] are shown in Table 1 while the collision diameter $\left(\sigma^{\mathrm{N}-\mathrm{N}}\right)$ and energy well depth $\left(\varepsilon^{\mathrm{N}-\mathrm{N}} / \mathrm{k}\right)$ for nitrogen are 3.615 $\AA$ and $101.5 \mathrm{~K}$, respectively. The interaction energy between sites $\mathrm{i}$ and $\mathrm{j}$ on two molecules $(\varphi \mathrm{ij})$ is calculated using the Lennard-Jones 12-6 equation [9].

$$
\varphi_{i j}=4 \varepsilon_{i j}\left[\left(\frac{\sigma_{i j}}{r_{i j}}\right)^{12}-\left(\frac{\sigma_{i j}}{r_{i j}}\right)^{6}\right]
$$

where $r_{i j}, \varepsilon_{i j}$ and $\sigma_{i j}$ are the distance between the two sites, the well depth of the interaction potential and the collision diameter of sites, respectively, and $k$ is Boltzmann's constant. Beside the interaction among LJ sites, the interaction between two charges takes the form of a Coulomb law of electrostatic interaction [9].

\begin{tabular}{|c|c|c|c|}
\hline parameter & value & parameter & value \\
\hline$\sigma^{c-c}$ & $2.757 \AA$ & $\varepsilon^{c-c} / \mathrm{k}$ & $28.129 \mathrm{~K}$ \\
\hline$\sigma^{0-0}$ & $3.033 \AA$ & $\varepsilon^{0-0} / \mathrm{k}$ & $80.507 \mathrm{~K}$ \\
\hline$q^{c}$ & $0.6512 e$ & $q^{\circ}$ & $-0.3256 \mathrm{e}$ \\
\hline$\lambda^{c-0}$ & $1.149 \AA$ & & \\
\hline
\end{tabular}

Table 1

Molecular parameters of the 3-center-LJ model

$$
\varphi_{q^{i} q^{j}}=\frac{1}{4 \pi \varepsilon_{0}} \cdot \frac{q^{i} q^{j}}{r_{i j}}
$$

where $\varepsilon_{0}$ is the permittivity of free space. 


\subsection{Solid model}

The solid model used in this study is the carbon-based adsorbents' structure whose pores typically have a slit-shaped geometry. A simple slit pore of finite length is modeled as a parallel pair of finite length walls which consist of graphite layers and perpendicular to the z-axis. Each of two walls consists of three graphene layers, and these layers are stacked on top of each other with an interlayer spacing of $3.354 \AA$. The width $\mathrm{H}$ of this slit pore model is defined as the distance between a plane passing through all carbon atom centers of the outmost layer of one wall and the corresponding plane of the other wall. The configuration of carbon atoms in each layer takes the form of condensation of aromatic rings of six carbon atoms. The adjacent carbon-carbon distance is $1.42 \AA$ [6]. Figure 1 shows the schematic diagram of a carbon pore, which is finite in length in the $x$ and $y$ directions [7]

Figure 1

Side view of one pore wall, comprising of three graphene layers.

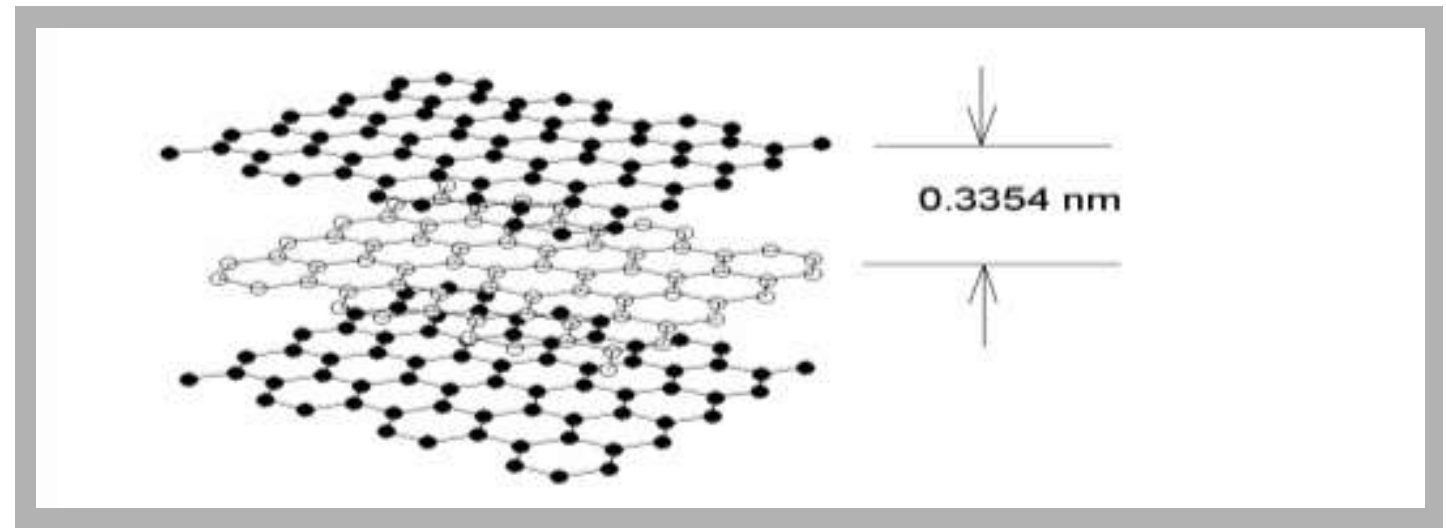

In this study all graphene layers are assumed to be square and equal in size, and they have a linear dimension of $60 \AA$, because it is reported in the literature [5] that the size of the graphene layer is between 20 and $70 \AA$. We choose values for pore width from 6.3 to $30 \AA$, to represent the micropore and mesopore in activated carbons. The LJ parameters for carbon, $\varepsilon_{s s} / \mathrm{k}$ and $\sigma_{\mathrm{ss}}$, are $28 \mathrm{~K}$ and $3.4 \AA$ respectively [2]. The interaction energy between a fluid molecule and a carbon atom is calculated by the Lennard-Jones 12-6 potential. These cross molecular parameters, $\sigma_{\mathrm{sf}}$ and $\varepsilon_{\mathrm{sf}}$ are calculated from the Lorentz-Berthelot rule.

\subsection{Simulation method}

We adopt the Metropolis algorithm in the Monte Carlo (MC) simulation [9] and the GCMC ensemble is used to obtain the adsorption isotherms of $\mathrm{N}_{2}$ and $\mathrm{CO}_{2}$. The simulation box for this ensemble is a finite length carbon slit pore, and has a linear dimension of $60 \AA$ in $\mathrm{x}$ and $\mathrm{y}$ directions. We assume that the top and the bottom of the simulation box are two walls of the slit pore, and each wall consists of three graphene layers. We specify the volume of the box (i.e. pore volume), the chemical potential and the temperature of the system to obtain the adsorption equilibrium. One GCMC cycle consists of one thousand displacement moves and attempts of either insertion or deletion with equal probability. For an adsorption branch of the isotherm 20,000 cycles are typically needed for the system to reach equilibrium, and additional 20,000 cycles are used to obtain ensemble averages. For each point on the adsorption branch, we use an empty box as the initial configuration, and the simulation is carried out until the number of particles in the box does not change (in statistical sense). The pressure of the bulk gas corresponding to a given chemical potential are calculated from the equation of state proposed by Johnson et al. [10].

\subsection{Experimental works}

The experimental data for $\mathrm{CO}_{2}$ and $\mathrm{N}_{2}$ adsorptions in $\mathrm{LAC}$ and $\mathrm{CAC}$ were obtained by using an Intelligent Gravimetric Analyzer (IGA) model IGA-002 supplied by Hiden Analytical Ltd., UK. Prior to adsorption experiments, the carbon sample $(0.12 \mathrm{~g})$ is outgassed at $200{ }^{\circ} \mathrm{C}$ for $10 \mathrm{hr}$ and then allowed to cool down to the adsorption temperature. Three types of LAC which differ in the percentage of burn-off are used in this study. 


\section{Results and discussions}

We shall start our discussion by presenting the experimental data for $\mathrm{CO}_{2}$ in these two types of activated carbon at 273 and $300 \mathrm{~K}$. Next we shall discuss the adsorption isotherms of $\mathrm{CO}_{2}$ at 273 and $300 \mathrm{~K}$ and that of $\mathrm{N}_{2}$ at $77 \mathrm{~K}$ in finite length pore with different widths to show the effects of pore width on the adsorption isotherm and then later the adsorption isotherm obtained by using the GCMC for $\mathrm{CO}_{2}$ at different temperatures will be compared. Finally the simulation results of $\mathrm{CO}_{2}$ and $\mathrm{N}_{2}$ in finite length pore and measured isotherm data will be used to characterize these activated carbon samples and compare with pore size distribution (PSD) determined by using nitrogen adsorption isotherms at $77 \mathrm{~K}$ and Density Functional Theory (DFT) method.

\subsection{Adsorption in activated carbon}

The adsorption isotherms of carbon dioxide obtained for CAC and LACs at 273 and $300 \mathrm{~K}$ are shown in Figures 2. The same behaviour can be observed the experimental data show a gradual increase in that of isotherm at low relative pressures. This is a typical isotherm observed for many micropore adsorbents, at relative pressure approaching unity; it may rise if large pores are present due to the capillary condensation in the mesopore. It is noted that the adsorption of $\mathrm{CO}_{2}$ is operated at pressure lower than the saturated pressure. The adsorption capacity decreases when the temperature increases. The data of nitrogen at $77 \mathrm{~K}$ are shown in Figure 3 as a sharp change at low relative pressures and then increase gradually at high relative pressures.
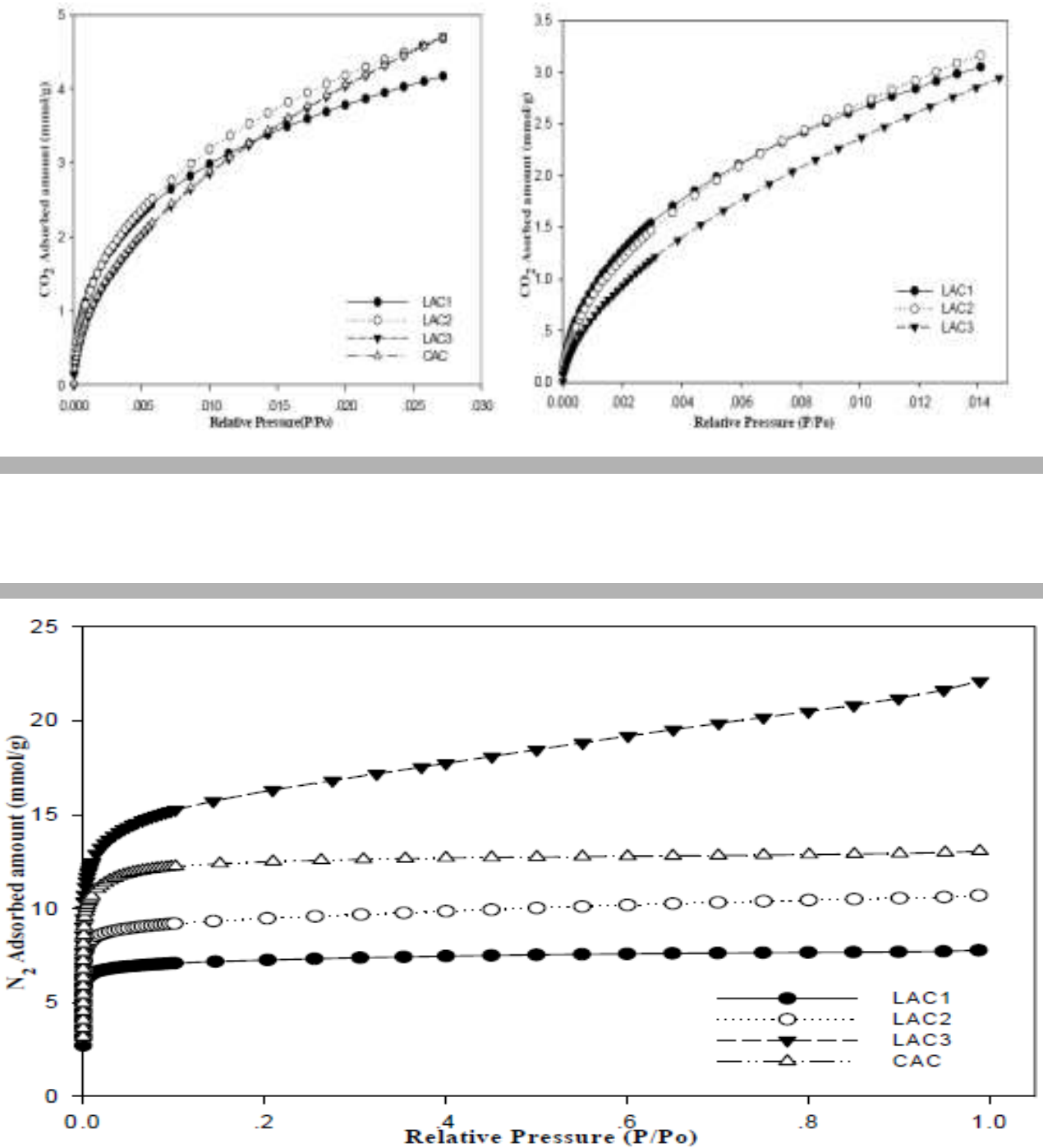

Adsorption isotherms of $\mathrm{CO}_{2}$ in LAC1, LAC2,

LAC3 and CAC at 273 (left) and $300 \mathrm{~K}$ (right).
Figure 3

Adsorption isotherms of $\mathrm{N}_{2}$ in LACs and $\mathrm{CAC}$ at $77 \mathrm{~K}$. 


\subsection{Simulation isotherms}

The simulated isotherms versus pressures for $\mathrm{CO}_{2}$ at 273 and $300 \mathrm{~K}$ and those for $\mathrm{N}_{2}$ at $77 \mathrm{~K}$ in single carbon slit pores of various pore widths (from 6.3 to $30 \AA$ ) up to the saturation vapour pressure are obtained by using the GCMC method, as shown in Figures 4 and 5 , respectively. The snapshots of either $\mathrm{CO}_{2}$ or $\mathrm{N}_{2}$ molecules inside the pores at various pressures are also shown in Figures 6 to study the adsorption behaviour of fluid in different pore widths. The simulation isotherms of $\mathrm{CO}_{2}$ in the slit pore model show the continuous pore filling of a single layer for the width less than 8.5 $\AA$. The isotherm drops with increasing of pore widths. This is due to the packing effect that leads to the difference in maximum density in each pore. The high maximum density of $6.3 \AA$ pore is due to the reason that $\mathrm{CO}_{2}$ particles can tightly fit inside the pore as a single layer. For pores larger than $8.5 \AA, \mathrm{CO}_{2}$ molecules can form a monolayer along the pore wall and then the additional layers next to the monolayer. The monolayer and pore filling mechanism can be observed for the larger pore widths of 20 to $27 \AA$, as one can see the slope of isotherms increases gradually due to the formation of monolayer and then it shows a small jump in adsorption isotherm at high pressures due to the pore filling behavior. The capillary condensation is not observed for these pores which differ from that observed for a spherical molecule of argon, nitrogen and methane. This is due to the fact that the 3-center-LJ model of $\mathrm{CO}_{2}$ forms a less dense packing while the spherical molecule forms a dense structure [1]. However the additional capillary condensation is observed in the case of adsorption in the larger mesopores.

The similar behavior is also observed for the simulation isotherms of $\mathrm{N}_{2}$ at $77 \mathrm{~K}$; however the clear capillary condensation is observed for the width larger than $17 \AA$ as shown in Figure 5 . This is due to the effects of the molecular structure as discussed in the previous section.

Figure4

Simulation results of $\mathrm{CO}_{2}$ in slit pores at (a) 273 and (b) $300 \mathrm{~K}$.
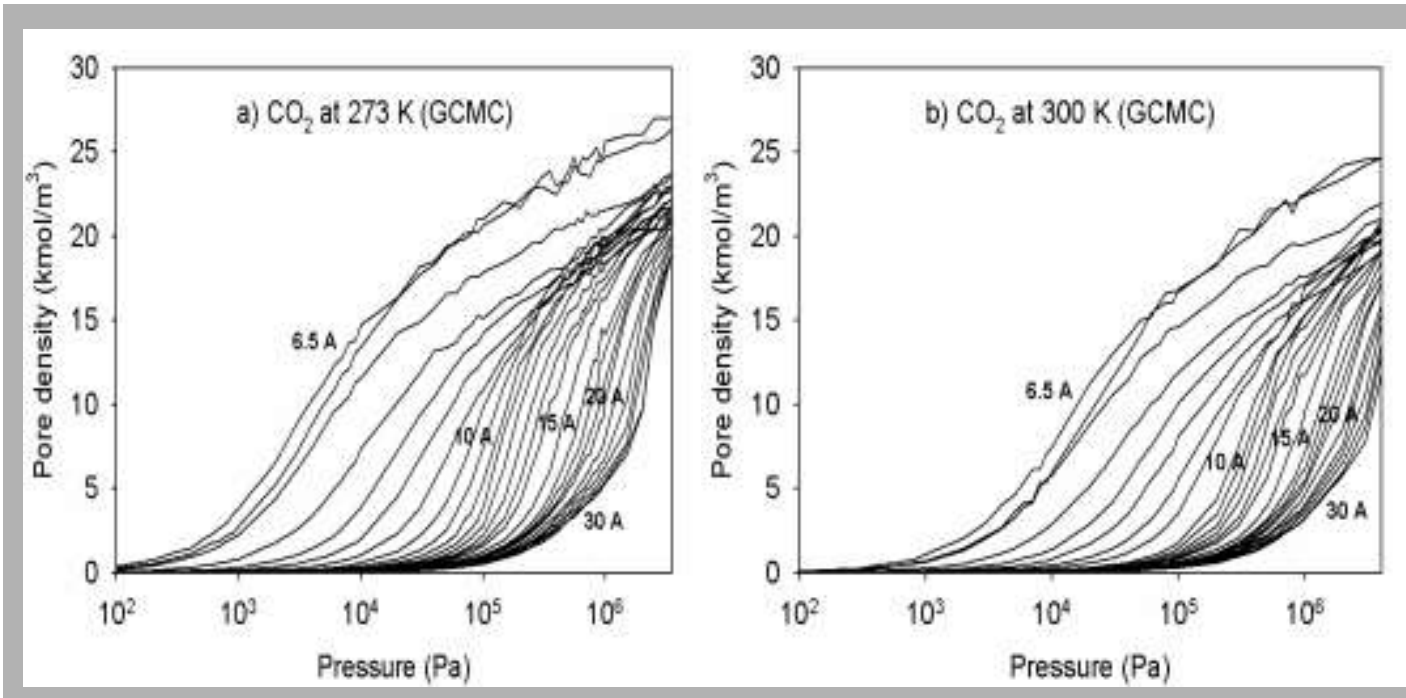

Figure 5

Simulation results of $\mathrm{N}_{2}$ in slit pores at $77 \mathrm{~K}$.

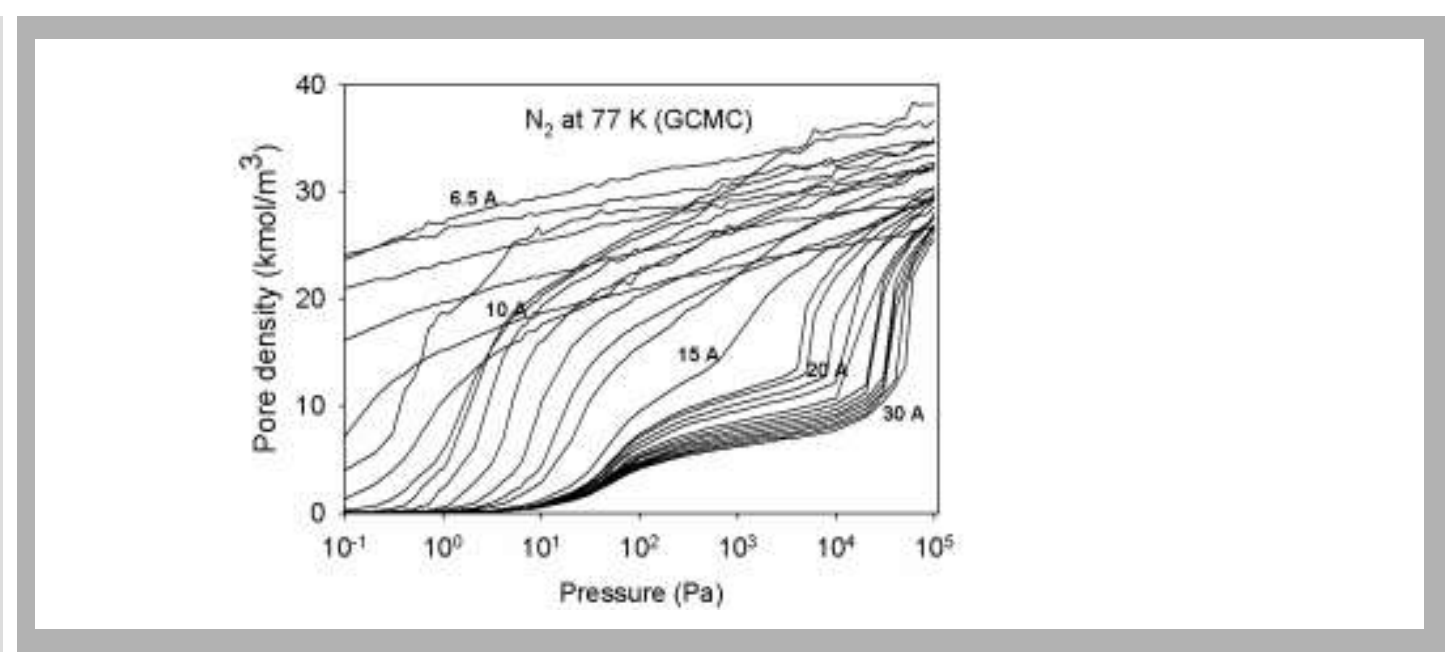




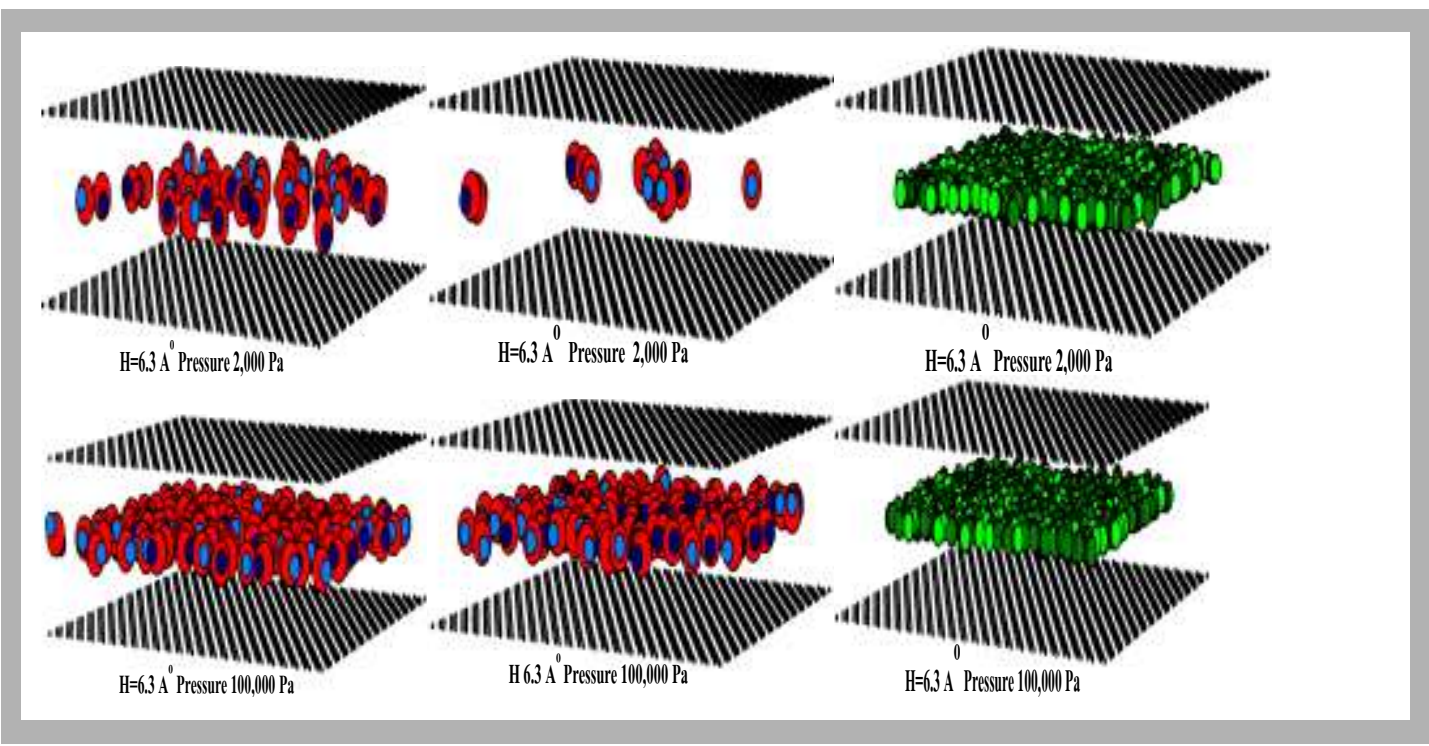

Figure 6a

Snapshots of $\mathrm{CO}_{2}$ at 273 and $300 \mathrm{~K}$, and $\mathrm{N}_{2}$ at $77 \mathrm{~K}$ in pore width of $6.3 \AA$ at various pressures
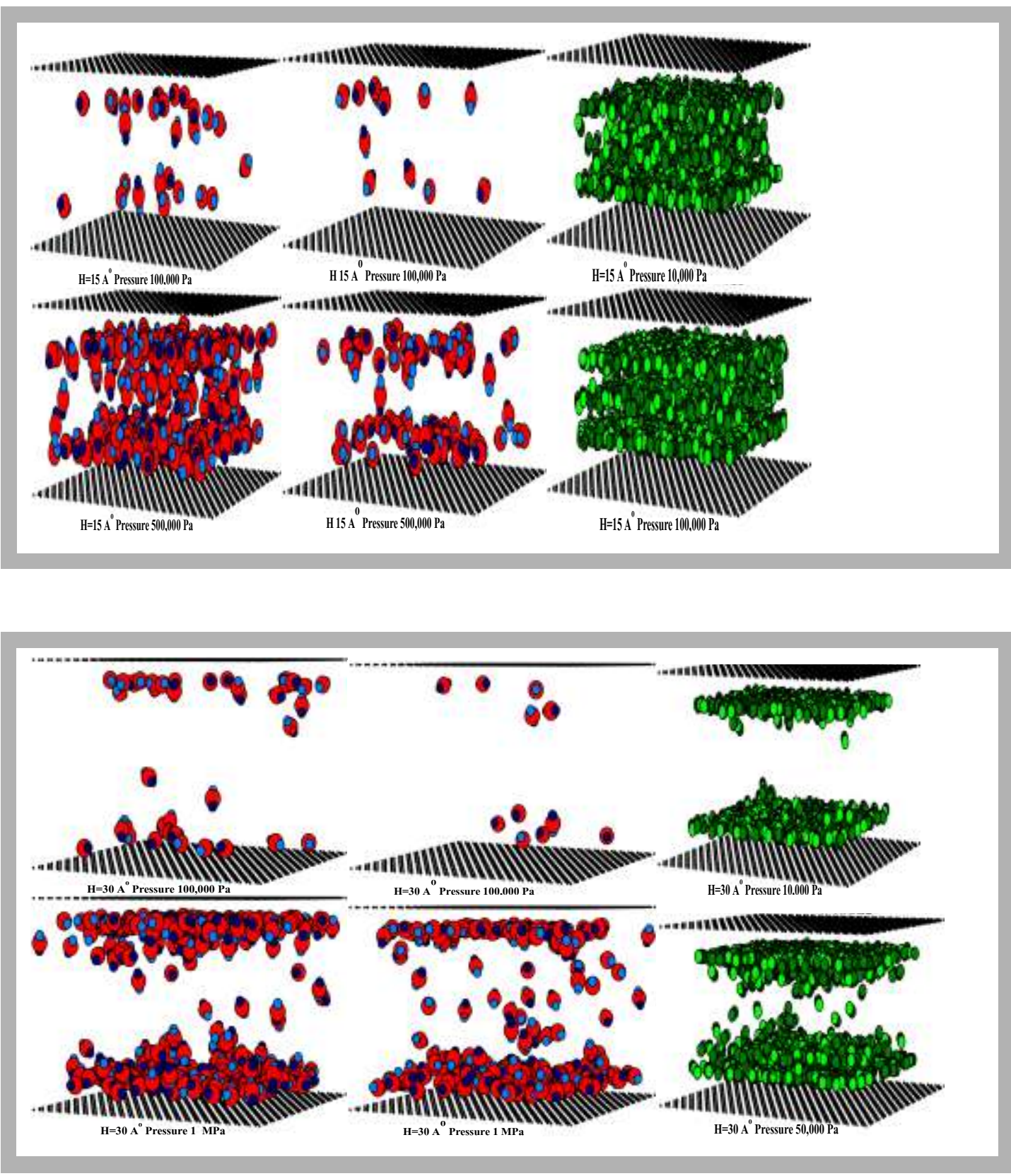

Figure 6b

Snapshots of $\mathrm{CO}_{2}$ at 273 and $300 \mathrm{~K}$, and $\mathrm{N}_{2}$ at $77 \mathrm{~K}$ in pore width of $15 \AA$ at various pressures.

Figure 6c

Snapshots of $\mathrm{CO}_{2}$ at 273 and $300 \mathrm{~K}$, and $\mathrm{N}_{2}$ at $77 \mathrm{~K}$ in pore width of $30 \AA$ at various pressures. 
The snapshots of $\mathrm{CO}_{2}$ molecules in the finite pores of $6.3,15.0$, and $30.0 \AA$ widths at 273 and $300 \mathrm{~K}$ and those of $\mathrm{N}_{2}$ at $77 \mathrm{~K}$ are shown in Figures 5 at various pressure values. In these figures, the black spheres represent carbon atoms of graphene layers, and for clarity we show only one graphene layer for each wall, while the red spheres represent nitrogen atoms. The number of layers in the 6.3, 15.0, and $30.0 \AA$ is 1, 4 and 8, respectively; however for the larger pores the higher layers are not quite clear due to that $\mathrm{CO}_{2}$ molecules can rotate in any directions.

It will be noted that the behavior in each pore is similar irrespective of its width, i.e. the adsorbed phase starts at low pressure by forming the two contact layers adjacent to the two walls due to the stronger solid-fluid interaction near the pore wall (except the $6.3 \AA$ width that has a single layer). When the pressure is increased, these contact layers are complete and the inner cores are then filled. In the larger pores, we can observe the presence of meniscus; the shape of meniscus is cylindrical and becomes flat at pressures close to the vapor pressure. It is noted that a study of meniscus is not possible with the simulation of infinite pores ${ }^{7}$. In the case of $\mathrm{CO}_{2}$ the snapshots also support that the greater adsorption occurs at lower temperature at the same pressure and width.

\subsection{Effects of temperature on the adsorption of $\mathrm{CO}_{2}$}

The adsorption isotherms of carbon dioxide at different temperatures (195.5, 273 and $300 \mathrm{~K})$ in semi-logarithmic scale obtained for three pore widths of 8,16 and $26 \AA$ are shown in Figures 7. What we have observed for the adsorption in different widths at 273 and $300 \mathrm{~K}$ is also observed for those at $195.5 \mathrm{~K}$. However the adsorption isotherm decreases with an increase of temperature which usually observed in the physical adsorption. This is due to that the adsorption is the exothermic process and the adsorbed molecules acquire the greater energy to evaporate [6].

Figure 7a Simulation isotherms of $\mathrm{CO}_{2}$ at different temperatures for pore width of $8 \AA$.
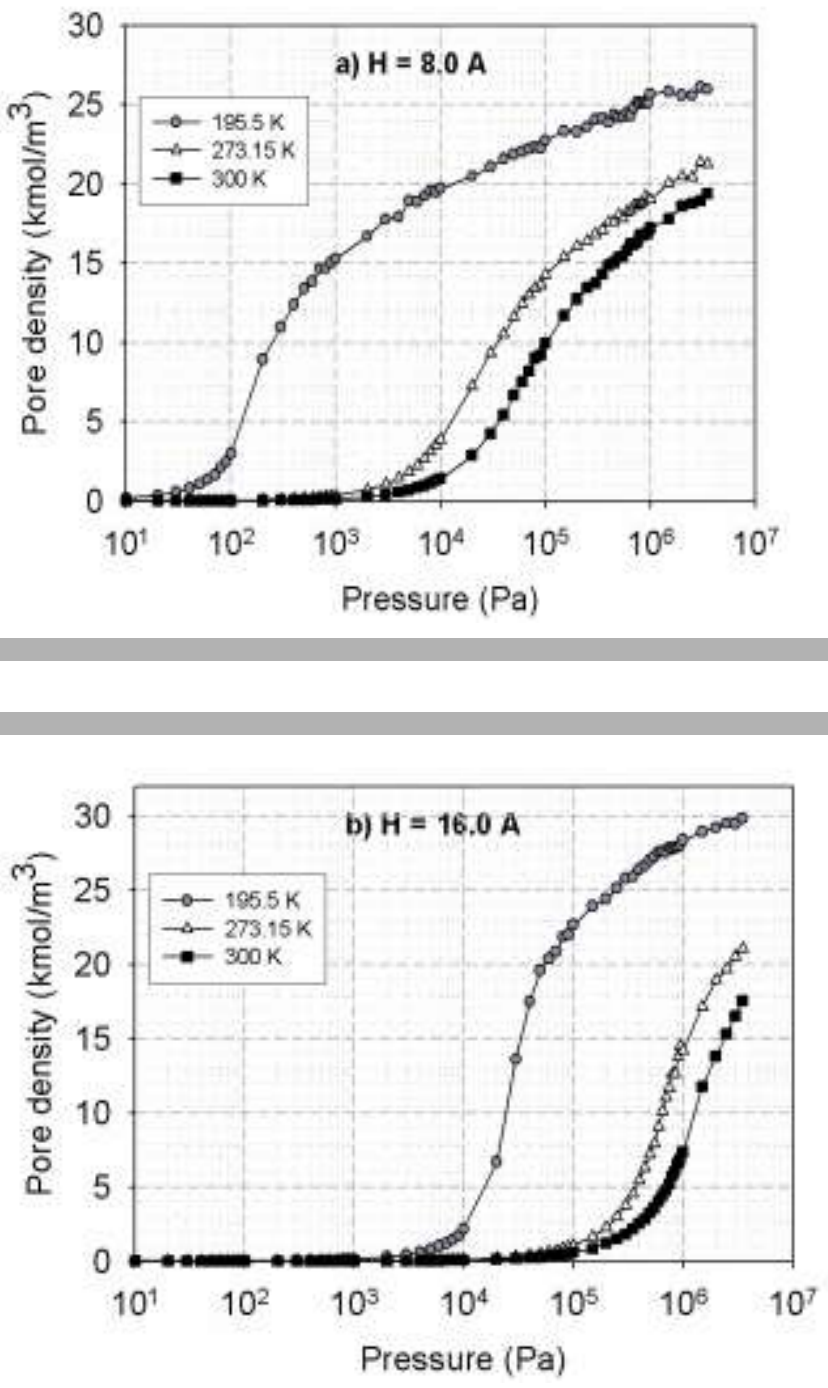


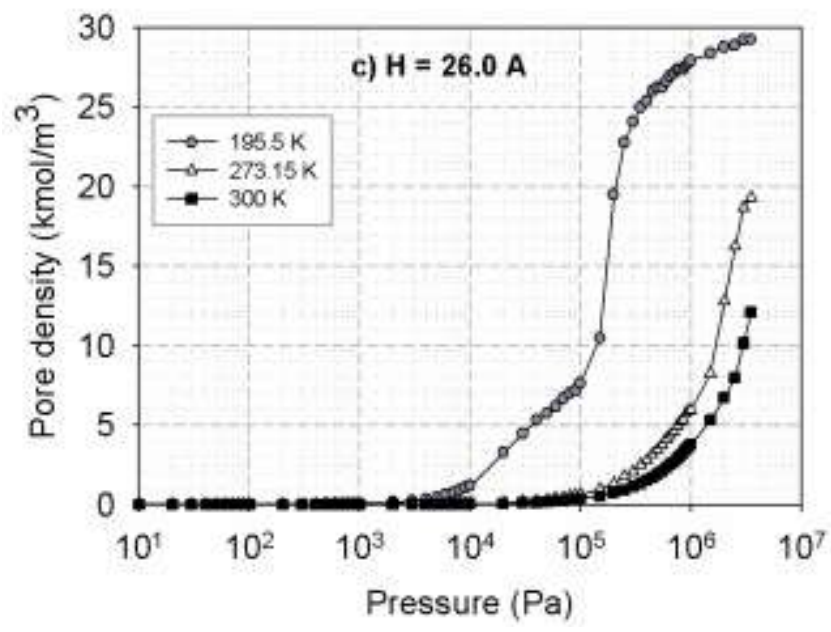

Figure 7c

Simulation isotherms of $\mathrm{CO}_{2}$ at different temperatures for pore width of $26 \AA$

\subsection{Pore size distribution (PSD) analysis.}

A method to determine the PSD developed based on GCMC simulations and measured isotherm data of $\mathrm{CO}_{2}$ proposed by Samios et al. [11] is used in this study. The set of simulation results are compared against the corresponding experimental isotherm counterpart by using an optimization function of MATLAB code. The PSD obtained by using adsorption isotherms of nitrogen at $77 \mathrm{~K}$ for CAC, LAC1, LAC2 and LAC3 are shown in Figures 8 . The total pore volume (micropore and mesopore) using the GCMC for CAC, LAC1, LAC2 and LAC3 is $0.395,0.221,0.315$ and $0.690 \mathrm{~cm}^{3} / \mathrm{g}$, respectively, which are similar to that of $0.45,0.27,0.37$ and $0.76 \mathrm{~cm}^{3} / \mathrm{g}$ using the DFT method for the corresponding carbons. However, the small pores of width less than $10 \AA$ can be observed in the case of using GCMC ensemble. The reconstructed isotherms from PSD function agree very well with the experimental data as shown in Figures 9.
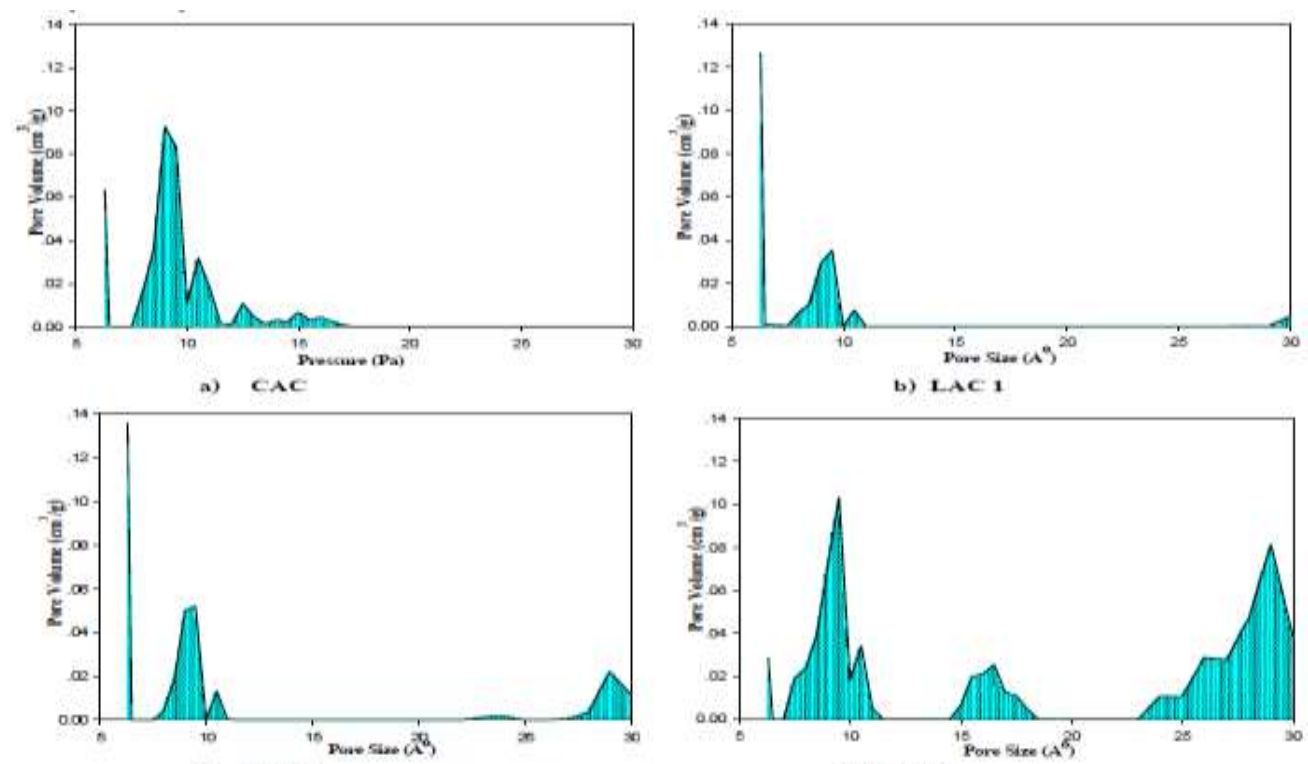

e) LAC 2

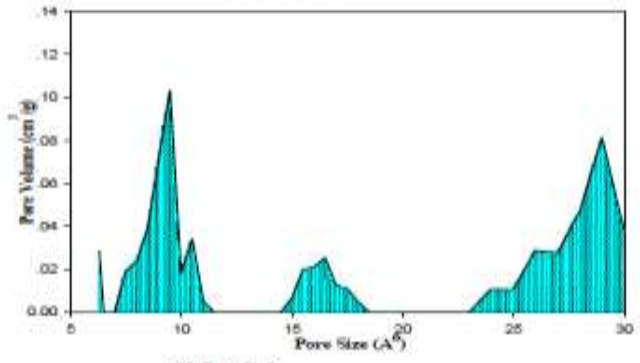

d) LAC $A$
Figure 8

Pore size distribution obtained for $\mathrm{CAC}$ and LACs using adsorption isotherms of $\mathrm{N}_{\text {, }}$ at $77 \mathrm{~K}$ 
Figure 9

Simulation isotherms and experimental data of $\mathrm{N}_{2}$ at $77 \mathrm{~K}$
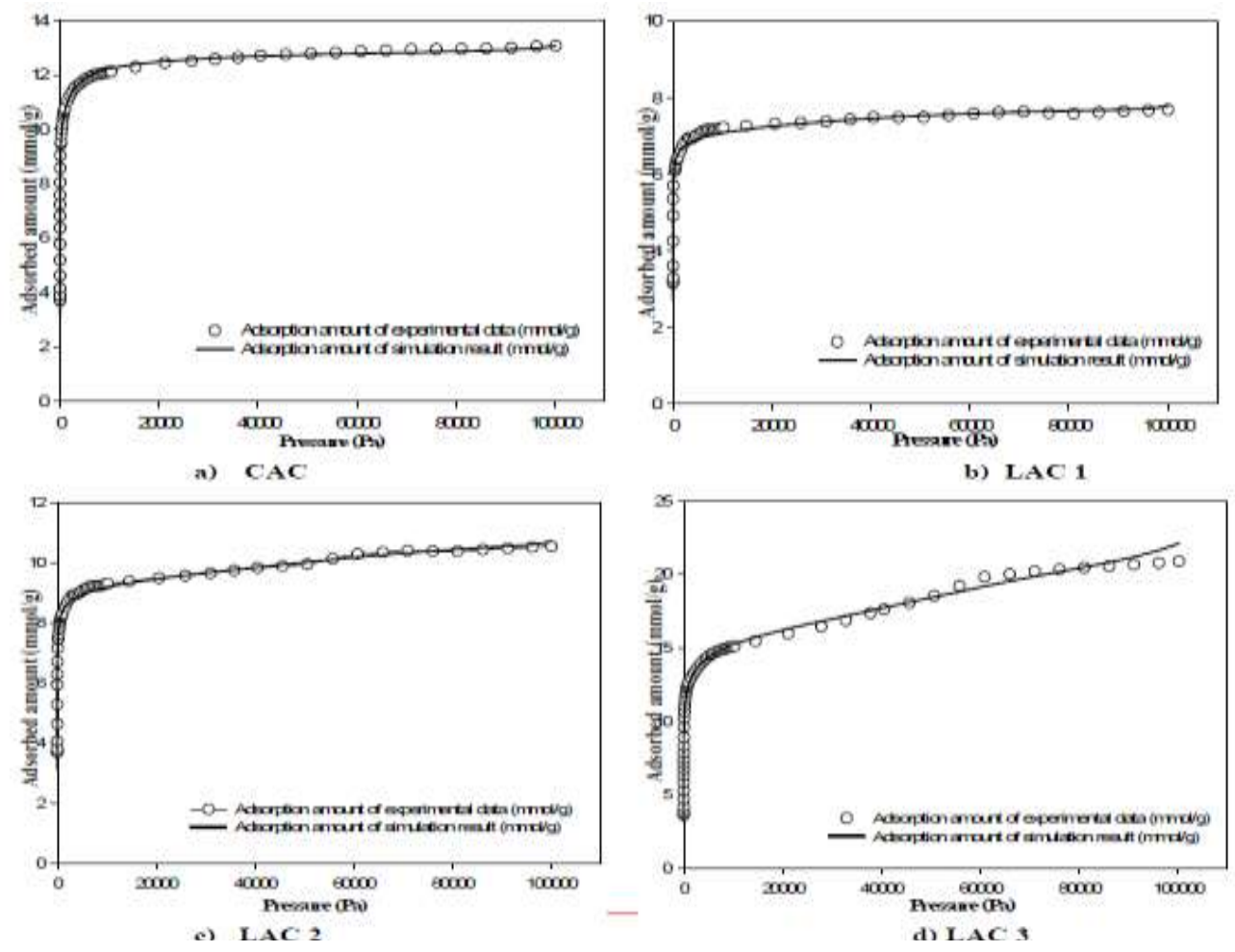

Having seen the PSD obtained from the $\mathrm{N}_{2}$ adsorption isotherm, now turn to the PSD obtained from that of $\mathrm{CO}_{2}$ at $273 \mathrm{~K}$ as shown in Figure 10. A specific pore volume (mostly micropore) obtained by $\mathrm{CO}_{2}$ adsorption for CAC, LAC1, LAC2 and LAC3 is $0.371,0.301,0.348$ and $0.366 \mathrm{~cm}^{3} / \mathrm{g}$, respectively. These values differ from those obtained from nitrogen isotherms at $77 \mathrm{~K}$ determined by a density functional theory (DFT) method. The micropore volume determined from DFT is $0.37,0.20$, 0.26 and $0.41 \mathrm{~cm}^{3} / \mathrm{g}$ for the corresponding activated carbons. The differences between these results may be due to that the 3-center-LJ model of $\mathrm{CO}_{2}$ does not completely fill in the larger pores because of its orientation requirement. It is reported in the literature [12] that the burn off percentage for LAC1, LAC2 and LAC3 is 19,26 and $60 \%$, respectively, the micropore and mesopore volumes increase with burn off. The micropore volume obtained from the GCMC results and experimental data for $\mathrm{CO}_{2}$ also increases with burn off for the Longan seed activated carbons. However it is greater than that obtained from the DFT method and experimental data for $N_{2}$. This may be due to that the collision diameter of carbon dioxide $\left(\sigma^{\mathrm{C}-\mathrm{C}}=2.757 \AA\right)$ is smaller than that of nitrogen $\left(\sigma^{\mathrm{N}-\mathrm{N}}=3.615 \AA\right)$, and this leads to the adsorption of $\mathrm{CO}_{2}$ in the small pore widths.

\section{Figure 10}

Pore size distribution obtained for CAC and LACs using the adsorption isotherms of $\mathrm{CO}_{2}$ at $273 \mathrm{~K}$.

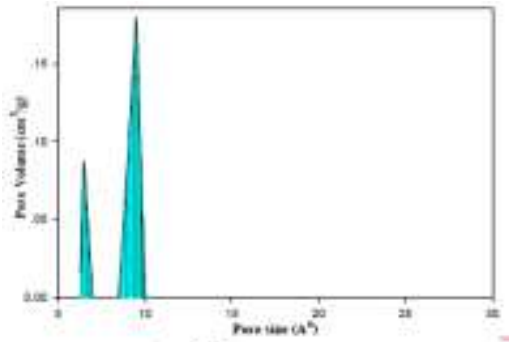

a) $\mathrm{CaC}$

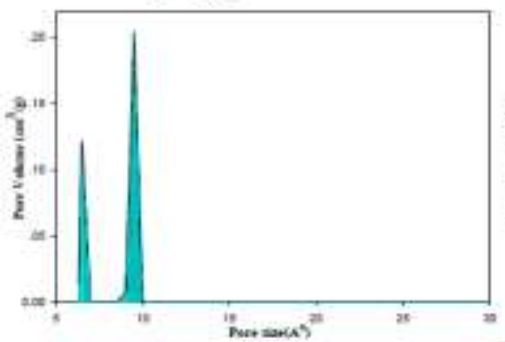

e) Lic:

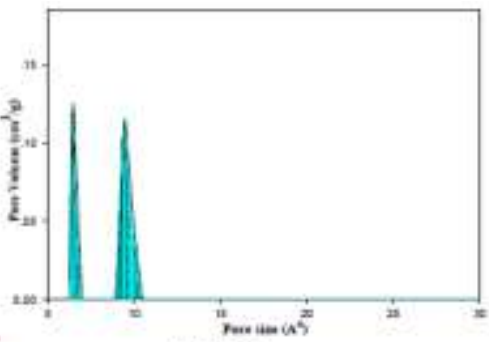

b) LAC 1

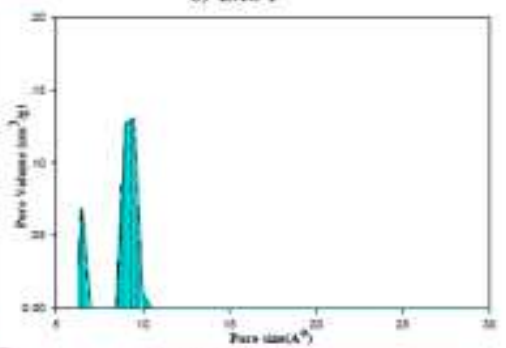

d) LAC 3 
The reconstructed adsorption isotherm obtained from the PSD of $\mathrm{CO}_{2}$ data agrees well with the experimental results for all types of activated carbon as shown in Figure 11. Therefore the $\mathrm{CO}_{2}$ data at low pressures can be used to determine the PSD for the micropore while the $\mathrm{N}_{2}$ adsorption isotherm is used to determine the total pore volume in activated carbon. For more accuracy, the PSD obtained from nitrogen isotherm should be used together with the PSD obtained from $\mathrm{CO}_{2}$ isotherm. The different probe and temperature can affect the evaluation of PSD of activated carbons.
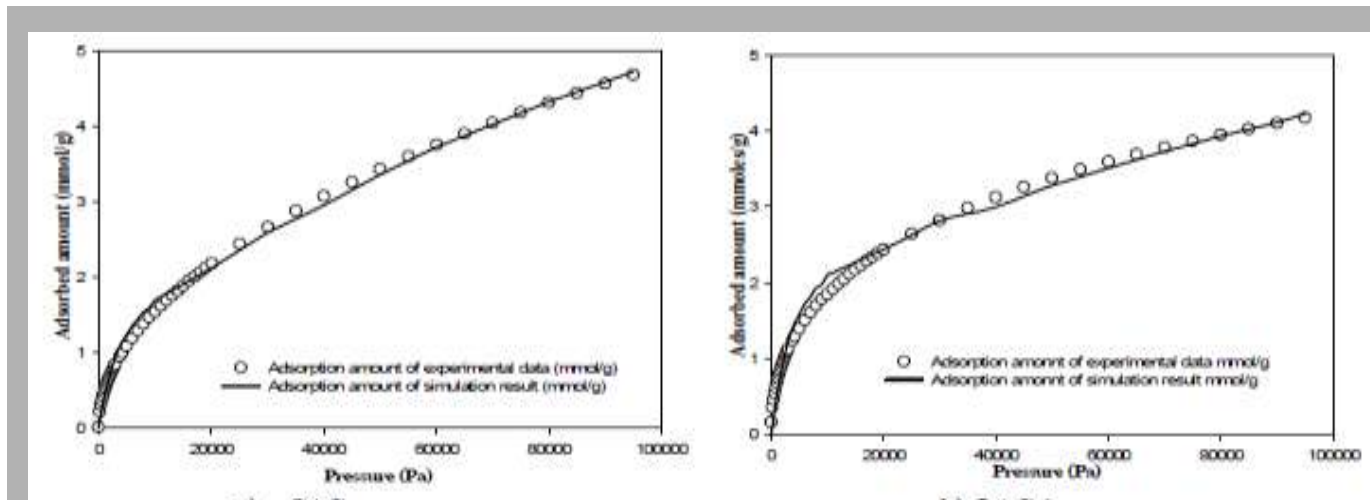

a) $\mathrm{CAC}$
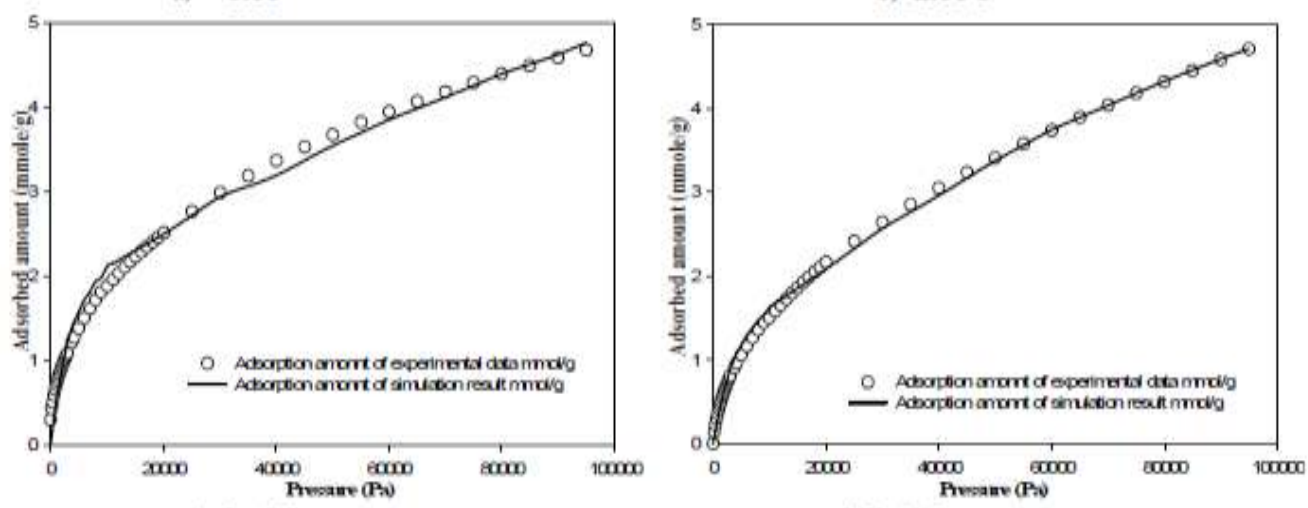

Figure 11

Simulation isotherms and experimental data c) $\mathrm{LAC} 2$

d) $\mathrm{LAC} 3$ of $\mathrm{CO}_{2}$ at $273 \mathrm{~K}$.

\section{Conclusions}

The adsorptions of $\mathrm{CO}_{2}$ and $\mathrm{N}_{2}$ in finite-length carbon slit pores have been presented in this paper. The adsorption behaviour and PSD function depends on the pore width, molecular probe and temperature. The GCMC simulation isotherms for different single pore width together with the experimental data can be used to determine the PSD that similar to that obtained by DFT method and the reconstructed isotherm is in a good agreement with the data. The adsorption data for carbon dioxide is suitable for characterization of porous solid with micropores. 


\section{ACKNOWLEDGEMENT}

We acknowledge School of Chemical Engineering and Institute of Research and Development, Suranaree University of Technology for financial support. 


\section{REFERENCES}

[1] P. I. Ravikovitch, A. Vishnyakov, R. Russo and A. V. Neimark, "Unified Approach to Pore Size Characterization of microporous Carbonaceous Materials from $\mathrm{N}_{2}$, Ar and $\mathrm{CO}_{2}$ Adsorption Isotherms," Langmuir, vol. 16, pp. 2311-2320, 2000.

[2] D. D. Do and H. D. Do, "Pore Characterization of Carbonaceous Materials by DFT and GCMC: A Review," Adsorption Science and Technology Journal, vol. 21, pp. 389-423, 2003.

[3] E. A. Muller, L. F. Rull, L. F. Vega and K. E. Gubbins, "Adsorption of Water on Activated Carbons: A Molecular Simulation Study," Journal of Physical Chemistry, vol. 100, pp. 1189, 1996.

[4] M. Maddox, D. Ulberg and K. E. Gubbins, "Molecular Simulation of Simple Fluids and Water in Porous Carbons," Fluid Phase Equilibria, vol. 104, pp. 145, 1995

[5] R. E. Franklin, "Crystallite growth in graphitizing and non-graphitizing carbons," Royal Society of London, vol. 209, pp. 196, 1951.

[6] D. D. Do, Adsorption Analysis: Equilibria and Kinetics, New Jersey, Imperial College Press, 1998.

[7] A. Wongkoblap, S. Junpirom and D. D. Do, "Adsorption of Lennard-Jones Fluids in Carbon Slit Pores of a Finite Length. A Computer Simulation Study," Adsorption Science and Technology Journal, vol. 23, pp. 1-18, 2005.

[8] J. G. Harris and K. H. Yung, "Carbon Dioxide's Liquid-Vapor Coexistence Curve and Critical Properties as Predicted by a Simple Molecular model," Journal of Physical Chemistry, vol. 99, pp. 12021-4, 1995.

[9] D. Frenkel and B. Smit, Understanding Molecular Simulation, $2^{\text {nd }}$ Ed." New York, Academic Press, 2002.

[10] J. K. Johnson, J. A. Zollweg and K. E. Gubbins, "The Lennard-Jones equation of state revisited," Molecular Physics Journal, vol. 78, pp. 591-618, 1993.

[11] S. Samios, A. K. Stubos, N. K. Kanellopoulos, R. F. Cracknell, G. K. Papadopoulos and D. Nicholson, "Determination of Micropore Size Distribution from Grand Canonical Monte Carlo Simulations and Experimental $\mathrm{CO}_{2}$ Isotherm Data," Langmuir, vol. 13, pp. 2795-2802, 1997.

[12] S. Junpirom, C. Tangsathitkulchai, M. Tangsathitkulchai and Y. Ngenyen, "Water adsorption in activatedf carbons with different burn-offs and its analysis using a cluster model," Korean Journal of Chemical Engineering, vol. 25, pp. 825-832, 2008. 